\title{
A INSERÇÃO DA RÉGUA DE CÁLCULO CIRCULAR COMO FERRAMENTA PARA O ENSINO DE LOGARITMO
}

\section{THE INTEGRATION OF THE CIRCULAR SLIDE RULE AS A TOOL FOR LOGARITHM TEACHING}

\author{
Verusca Batista Alves ${ }^{1}$ \\ Universidade Estadual do Ceará - UECE \\ Hosana de Fátima Melo da Silva \\ Universidade Estadual do Ceará - UECE \\ Ana Carolina Costa Pereira ${ }^{3}$ \\ Universidade Estadual do Ceará - UECE
}

\section{Resumo}

A inserção da história no ensino de Matemática já é alvo de pesquisa entre estudiosos brasileiros. Nessa vertente, a utilização de instrumentos matemáticos que, historicamente foram importantes no desenvolvimento da Ciência, é uma forma de compreender um determinado assunto. Dentre eles, encontra-se a Régua de Cálculo Circular, um instrumento criado por William Oughtred (1574-1660) que incorpora conhecimentos matemáticos que implicam até os dias atuais. Em meio as possibilidades de uso desse instrumento, escolhemos tratar do conteúdo Logaritmos. Desta forma, este artigo discute brevemente sobre as potencialidades e limitações do uso da Régua de Cálculo Circular como ferramenta auxiliadora no ensino e na aprendizagem de Logaritmos a partir da visão de discentes de um curso de Licenciatura em Matemática da Universidade Estadual do Ceará - UECE. O curso teve duração de 34h/a, na qual por meio de questionários e atividades, obtivemos os dados pertinentes à pesquisa. De um modo geral, percebe-se que os discentes participantes não haviam tido contato com os instrumentos históricos anteriormente, muito embora já haviam trabalhado com a História da Matemática, mas que, após o curso, consideraram a utilização desses objetos uma forma de facilitar o ensino e a aprendizagem do conteúdo de Logaritmos. Fica claro que a utilização de meios alternativos na Educação Matemática favorece a compreensão dos assuntos estudados. Tem-se na História da Matemática, em particular a construção de instrumentos matemáticos, um meio para contribuir para um ensino mais significativo ao proporcionar uma visão diferenciada no ensino dos Logaritmos por meio da manipulação e análise do objeto.

Palavras-chave: Instrumentos matemáticos. História da Matemática. Formação inicial de professores. Régua de Cálculo Circular.

\section{Abstract}

The integration of the history in the teaching of Math is already a target of research among

\footnotetext{
${ }^{1}$ Email: verusca.alves@aluno.uece.br

${ }^{2}$ Email: hosana.melo@aluno.uece.br

${ }^{3}$ Email: carolina.pereira@uece.br
} 
Brazilian scholars. In this section, the use of mathematical instruments that historically were important in the development of Science is a way of understanding a given subject. Among them, there is the Circular Slide Rule, an instrument created by William Oughtred (1574-1660) that incorporates math skills which involve up to the present day. Among the possibilities of using this instrument, we chose to approach the content Logarithms. Therefore, this article discusses briefly on the potentialities and limitations of the use of the Circular Slide Rule as a helper tool in the teaching and learning Logarithms from the vision of students from an undergraduate course in Math at the States University of Ceará - UECE. The course lasted 34h/a, which by means of questionnaires and activities, we obtained the relevant data to the survey. In general, the participants students had not had contact with historical instruments previously, although they had already worked with the History of Math, but after the course, they considered the use of these objects as a way to facilitate the teaching and learning the contents of Logarithms. It is clear that the use of alternative means in Math Education promote understanding of subjects studied. It has been in the History of Math, in particular the construction of mathematical instruments, a way to contribute to a meaningful education to provide a differentiated view on the teaching of Logarithms by means of the manipulation and analysis of the object.

Keywords: Mathematical instruments. History of Mathematics. Initial teachers training. Circular Slide Rule.

\section{Introdução}

A História da Matemática é considerada por diversos autores, que discutem à formação de professores, como um recurso didático que pode auxiliar na aprendizagem da Matemática.

O uso de instrumentos matemáticos históricos é uma forma de estabelecer uma ligação entre o ensino de Matemática e a História. Entende-se que uma atividade prática e exploratória envolvendo a construção e o manuseio de um desses instrumentos pode facilitar a compreensão dos conteúdos relacionados ao mesmo. Saito $(2014$, p. 28) ressalta que:

\footnotetext{
As potencialidades didáticas e/ou pedagógicas na reconstrução de Instrumentos antigos podem ser exploradas por meio de uma proposta que busque revelar não só os conhecimentos matemáticos incorporados nesses Instrumentos, mas também a complexa rede de conhecimentos que "esteve" e "está" presente no processo de sua construção e uso.
}

Por isso, considera-se que a inserção da História da Matemática no ensino pode agregar conhecimentos importantes que vão além da construção de conceitos e que pode mobilizar a aprendizagem dos alunos.

Para que o professor possa trabalhar com instrumentos matemáticos históricos é necessário que o mesmo tenha conhecimento sobre esses, tanto no que se refere a sua história quanto ao conteúdo matemático envolvido. Atividades que agregam a 
manipulação de um instrumento envolvem a experimentação e " [...] facilita que o aluno levante hipóteses, procure alternativas, tome novos caminhos, tire dúvidas e constate o que é verdadeiro, válido, correto ou solução" (LORENZATO, 2006, p. 72).

Mendes (2008, p. 40) ressalta que:

[...] é adequado o uso de atividades que favoreçam a interatividade entre o sujeito e o seu objeto de conhecimento, sempre em uma perspectiva contextualizadora que evidencie três aspectos do conhecimento: o cotidiano, o escolar e o científico, principalmente quando são rearticulados ao longo do processo de manuseio de qualquer componente da atividade (o material manipulativo, as orientações orais e escritas e o diálogo estabelecido durante todo o processo ensino-aprendizagem, etc).

Portanto, o uso de um instrumento matemático histórico para o ensino, quando trabalhado em sala de aula por meio de atividades individuais e/ou em grupos, pode tornar o aprendizado mais eficiente.

Sabe-se que por muitas vezes os alunos apresentam dificuldade em compreender o assunto estudado, tornando a Matemática difícil para os mesmos. Ao lecionar os conteúdos com um instrumento matemático histórico, o professor empregará em sala de aula a História da Matemática, e de acordo com a metodologia utilizada, pode auxiliar para um melhor entendimento do assunto.

Este trabalho apresenta os resultados de um curso de extensão universitária sobre o uso da Régua de Cálculo Circular, sob o olhar do futuro professor de Matemática, observando-a como estratégia para o ensino de Logaritmos. Assim como, expõe um breve passeio histórico sobre a origem dos Logaritmos e sua relação com a Régua de Cálculo Circular e uma discussão sobre seu uso como recurso didático ressaltado pelos participantes do curso.

\section{Um passeio histórico sobre a Régua de Cálculo}

O século XVII sofreu diversos avanços em várias áreas do conhecimento, em particular na Matemática. Um deles foi a criação dos Logaritmos que mudou a vida de Astrônomos e Matemáticos, reduzindo o tempo gasto com operações de multiplicação e divisão extensas utilizadas em cálculos. Era costumeiro simplificar problemas envolvendo produto e divisão transformando-os em soma e subtração. Atualmente, conhecemos esse processo como: o logaritmo do produto é a soma dos logaritmos dos fatores e o logaritmo do quociente é a diferença do logaritmo do dividendo pelo do divisor. 
Historicamente, um dos instrumentos criados para tentar sistematizar essas operações foi a Régua de Cálculo Circular, que se utiliza das escalas Logarítmicas criadas por Edmund Gunter (1581-1626) a partir dos estudos realizados por John Napier (15501617). Segundo Pereira (2015, p. 41):

\begin{abstract}
A "Escala de Gunter" é uma linha reta, com os números dispostos de 1 a 10 de uma extremidade à outra, de tal forma que as distâncias ao longo da linha não são proporcionais aos números nele, mas sim os logaritmos dos referidos números. Essas escalas eram dispostas em uma régua de madeira em que cada uma das linhas dava logaritmos de funções trigonométricas. Sua utilização ainda agregava um par de compassos que marcavam adição e subtração das distâncias na escala de acordo com as propriedades dos logaritmos, ou seja, o produto e o quociente dos números.
\end{abstract}

A escala de Gunter foi, por muito tempo, confundida com a Régua de Cálculo Linear de William Oughtred (1574-1660), que por sua vez é outro modelo de régua que tinha propósitos semelhantes ao modelo circular. Porém, a diferença entre a régua linear e a escala de Gunter é que a primeira continha uma peça deslizante que facilitava obter os resultados dos cálculos.

Napier elaborou um sistema que chamou de Rabdologiae, que ficou conhecido como Barras de Napier ou Ossos de Napier, e realizava cálculos de multiplicações e divisões utilizando varetas. Por isso, é provável que Oughtred tenha se baseado na criação de Napier para construir suas réguas. Pereira (2015, p. 44) ainda afirma que:

William Oughtred foi o primeiro inventor de Réguas de Cálculo, embora não seja o primeiro a publicar esse Instrumento. Ele inventou as Réguas de Cálculo em 1622, mas as descrições desses Instrumentos só foram disponibilizadas para a imprensa a partir de 1632. Esse fato possibilitou, por algum tempo, nomear Richard Delamain (1600 - 1644), aluno de Ougthred, inventor da Régua de Cálculo Circular, porém sabemos que isso ocorreu de forma independente.

Como mencionado por Pereira (2015), Oughtred criou as réguas por volta de 1622, entretanto a descrição da régua em seu modelo circular só foi publicada entre 1632 e 1633 no livro Circles of Proportion and the Horizontal Instrument. Essa edição foi transcrita em latim por Oughtred e traduzida para o inglês por seu aluno William Foster, em 1633. $\mathrm{Na}$ obra o autor "descreve os princípios das escalas logarítmicas e o uso da régua de cálculo em uma ampla gama de aplicações, incluindo astronômico e de navegação" (PEREIRA, 2015, p. 49). A simbologia log para se referir aos Logaritmos também é creditada a Oughtred.

A Régua de Cálculo Linear (Figura 1) constitui-se de duas réguas deslizantes que 
possuem escalas Logarítmicas. A ideia implícita é transformar produto e quociente, respectivamente, em soma e subtração.

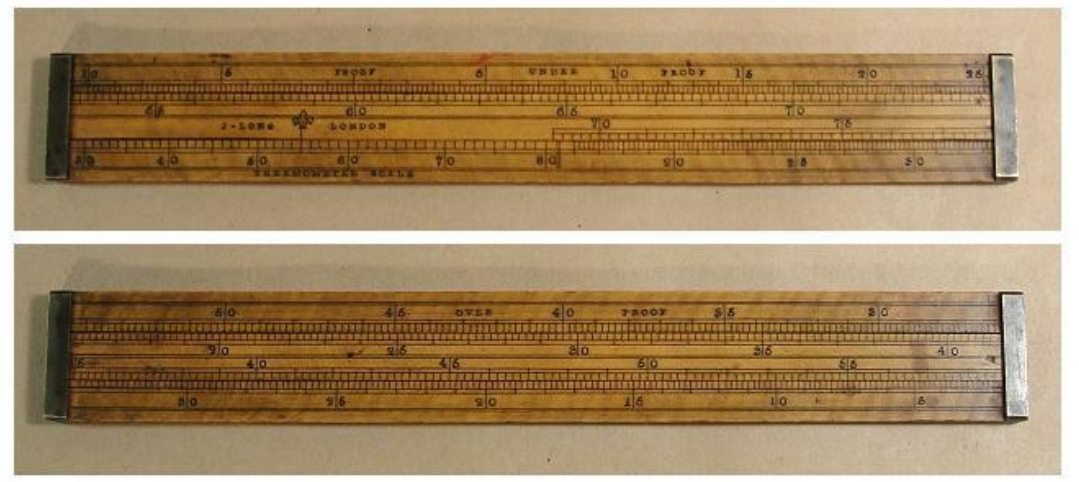

Figura 1 - Exemplo de uma Régua de Cálculo Linear datada de 1840

Fonte: http://collectingme.com/computing/Two_Sides_One_Slide_Alcohol_Slide_Rule

Outro modelo que podemos encontrar, é a Régua de Cálculo Circular (Figura 2).

Este exemplar apresenta suas marcações compiladas em uma circunferência, utilizando as escalas logarítmicas para efetuar cálculos de números extensos. Sua descrição trata-se de um disco com um ou dois cursores que se movimentam para efetuar os cálculos desejados.

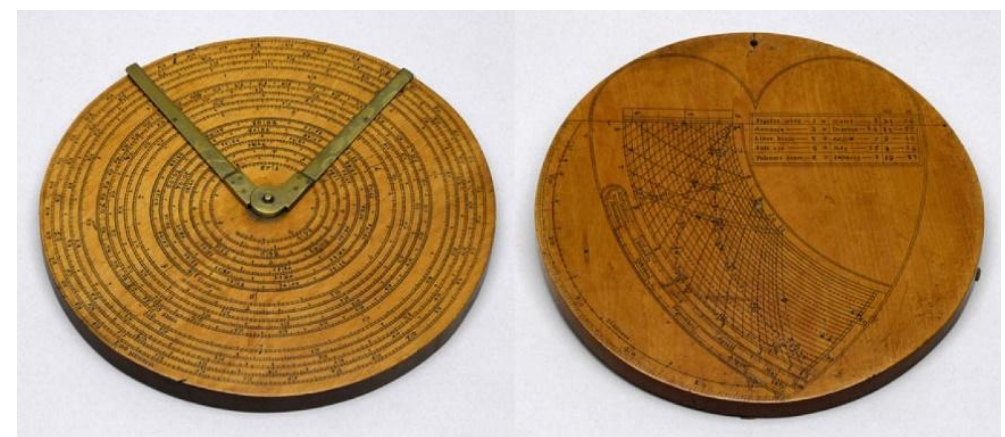

Figura 2 - Régua de Cálculo Circular, 1660-1680

Fonte: http://www.sciencemuseum.org.uk/images/I067/10328277

Antigamente as réguas eram muito utilizadas por Cientistas e Engenheiros em seus projetos, porém, com a criação das calculadoras, esse tipo de aparato entrou em desuso. A criação dos Logaritmos permitiu facilitar o cálculo de operações aritméticas reduzindo as operações para um grau de dificuldade inferior, como multiplicação em adição, divisão em subtração, potenciação em multiplicação e radiciação em divisão. Por isso, o estudo da construção matemática e física das réguas de cálculo pode ser um meio para complementar o estudo dos Logaritmos na Educação Básica. 


\section{A Régua de Cálculo Circular como recurso didático}

O instrumento matemático histórico é um recurso didático que pode facilitar tanto o ensino quanto a aprendizagem, pois oportuniza a verificação de resultados por meio do manuseio. Entretanto, pode-se questionar a respeito de como inserir esse instrumento em sala de aula. Em que momento o docente pode fazer uso do instrumento? Qual a melhor forma de inserir a Régua de Cálculo Circular na aula de Matemática?

Alguns dos estudos realizados sobre a Régua de Cálculo priorizam a parte matemática envolvida na construção da mesma utilizando conceitos atuais de Logaritmos. Um exemplo é o trabalho de Maia (2013), que apresenta atividades relacionadas ao funcionamento da Régua de Cálculo Linear associado às propriedades dos Logaritmos estudadas pelos alunos do primeiro ano do Ensino Médio. Porém, não faz uma discussão didática desse recurso.

O texto de Pereira e Oliveira (2013) propõe o uso pedagógico de um artefato histórico originado da criação e uso dos Logaritmos, no caso a Régua de Cálculo Linear, propondo atividades relacionadas ao estudo dos Logaritmos no Ensino Básico.

As dissertações de Pippa (2014) e Tanonaka (2008) traçam a história da Régua de Cálculo, sendo que a primeira propõe uma sequência didática para a utilização da régua em seu modelo Linear. Fonseca e Pereira (2015) fazem uma tentativa de inserção da Régua de Cálculo Linear no ensino, utilizando duas réguas deslizantes graduadas por meio dos logaritmos de base 10 com o objetivo de realizar multiplicações e divisões.

Um exemplo prático da utilização das Réguas no ensino pode ser visto no trabalho de Portela, Florêncio e Martins (2014), que apresenta uma proposta de manuseio da Régua de Cálculo Circular com a finalidade de indicar de maneira precisa e prática, qual a melhor escolha de combustível para um carro flex, considerando a variação dos preços do álcool e da gasolina.

Os trabalhos apresentados vislumbram uma possibilidade de aplicação no ensino, seja na formação inicial de professores ou com alunos da Educação Básica. Nesta etapa, vários obstáculos devem ser ultrapassados, dentre eles, tentativas e erros por parte dos alunos. Tais "erros de nossos alunos podem ser interpretados como verdadeiras amostragens dos diferentes modos que os alunos podem utilizar para pensar, escrever e agir.” (LORENZATO, 2006, p. 50). Assim, a experimentação é favorável, pois se trata de descobertas feitas pelos próprios alunos, em que eles investigam por meio de tentativas 
até obter sucesso.

A régua linear e a circular, dependendo da finalidade esperada pelo professor, consistem em um excelente recurso didático para a aula de Matemática, porém, o modelo circular possui algumas vantagens. Ela realiza operações sucessivas através do processo de somas de parcelas ou produto de fatores até obter o resultado desejado.

A régua circular também usa de princípios matemáticos adquiridos ao longo do processo de construção do conhecimento matemático que transpassam subáreas como a Álgebra e a Geometria. Como por exemplo, na sua construção, Oughtred possivelmente usou princípios geométricos para construir uma régua que realiza operações aritméticas.

Desta forma, a régua constitui-se como uma via que possibilita a ligação entre a História da Matemática e o conteúdo lecionado no Ensino Médio. Entretanto o professor deve considerar a necessidade da turma e do assunto abordado. A seguir, encontram-se duas propostas de inserção da Régua de Cálculo:

- O professor pode confeccionar o instrumento para todos os alunos e na sala de aula, os alunos podem manipulá-lo e descobrir as propriedades matemáticas por trás do artefato;

- O professor pode construir o instrumento em conjunto com os alunos, tanto a parte de marcações da régua quanto sua parte física, retornando as origens da Matemática do século XVII, para posteriormente aplicá-lo e verificar a utilização do artefato.

A construção e manuseio da régua de cálculo, em seus aspectos gerais, possibilitam ao professor usar métodos diferenciados para ensinar os conteúdos pretendidos, tais como realizar operações aritméticas, estudar o conceito de Logaritmo e comprovar a eficiência do instrumento por meio das propriedades dos logaritmos.

Uma sugestão para o uso da régua circular em sala de aula, é o professor apresentar o método de multiplicar e dividir sem indicar qual conteúdo e propriedade matemática está sendo utilizado. O objetivo é os alunos identificarem as propriedades dos Logaritmos, possibilitando a experimentação com a régua, e em seguida, a verificação da sua veracidade na calculadora científica.

Também, pode ser proposta como atividade a construção de uma régua, contendo os conhecimentos matemáticos lecionados ao longo do $1^{\circ}$ ano do Ensino Médio. O professor será o mediador, enquanto os alunos constroem e apresentam a régua confeccionada aos seus colegas, proporcionando uma interatividade entre a turma. 


\title{
Percepções do uso da Régua de Cálculo Circular no ensino: vislumbrando uma prática
}

A experimentação no ensino de Matemática apresenta a vantagem por perpassar a possibilidade de concretizar algo aplicável nas nuances de uma determinada experiência planejada e executada para um objetivo específico do estudo. Segundo Frota (2005, p. 2):

\begin{abstract}
Uma experiência Matemática é, por outro lado, coletiva, cultural e política uma vez que é vivenciada por pessoas com uma história, inseridas numa cultura, parte de uma comunidade. Essa história possibilita experiências distintas e a cada tipo de experiência Matemática é possível atribuir valorações diferenciadas, que podem, por vezes, vir carregadas de preconceitos e visões ideológicas.
\end{abstract}

Nesta perspectiva, planejou-se e aplicou-se o curso, de extensão universitária, intitulado $O$ uso de artefatos históricos para o estudo de Matemática: a Régua de Cálculo Circular como instrumento mediador no ensino dos Logaritmos com o intuito de apresentar o estudo do Logaritmo através de um instrumento, proporcionando uma reflexão sobre o uso da História da Matemática na Educação como um recurso para as aulas.

A proposta foi desencadeada nos meses de março e abril de 2016 num total de 34 horas-aulas para 20 licenciandos (Figura 3) do curso de Matemática da UECE. A coleta de dados se deu pela gravação de áudio das aulas, registros fotográficos, questionários (inicial e final) e atividades desenvolvidas ao decorrer do curso.

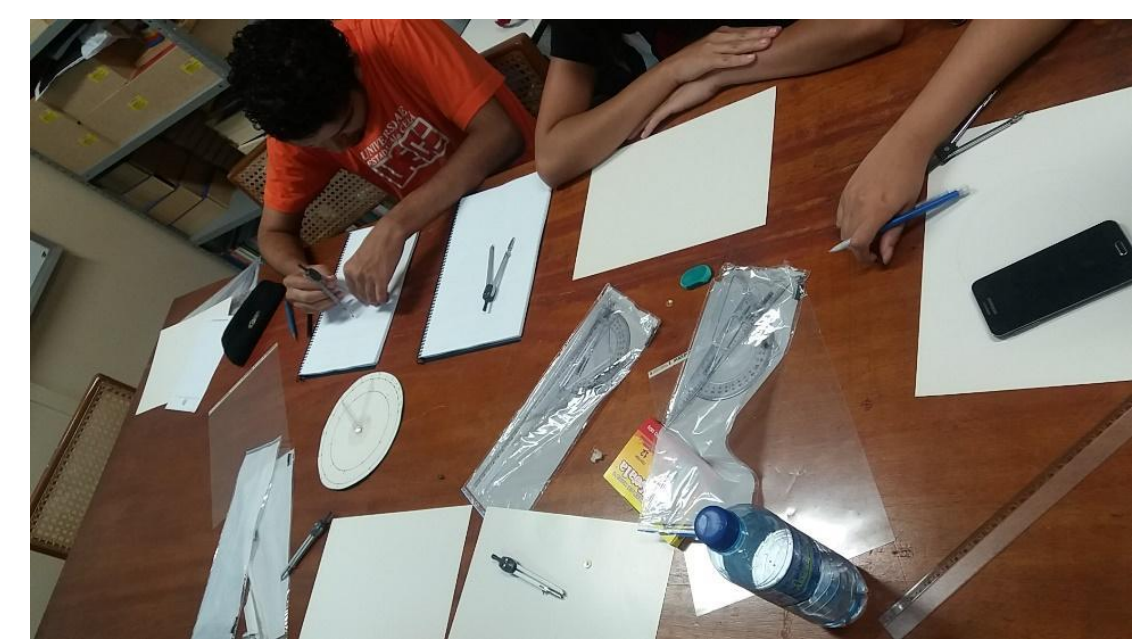

Figura 3 - Processo de construção da régua de cálculo circular no curso de extensão Fonte: Das autoras

A programação foi pensada para que o participante tivesse uma visão geral das 
possibilidades de inserção de instrumentos matemáticos na Educação Básica e o estudo efetivo da construção física e matemática da Régua de Cálculo Circular.

Assim, durante o curso, foi tratado de conteúdos metodológicos como, a História da Matemática como uma estratégia didática e a utilização de artefatos históricos para a compreensão de conceitos matemáticos. Também foi exposto um panorama histórico dos séculos XV e XVI a fim de mostrar a influência do período para o desenvolvimento da Ciência e a Matemática produzida na época. Além disso, uma breve revisão do conteúdo matemático de Logaritmos e da história de criação da régua de cálculo. Para finalizar, foi proposta uma atividade de construção do instrumento e uma discussão a respeito da utilização em sala de aula.

Dentre as várias discussões no decorrer do curso, a que envolveu as potencialidades, como por exemplo, a complementação do estudo do conteúdo Logaritmos, o desenvolvimento histórico da Matemática e as limitações, como a confecção e montagem do instrumento, proporcionou uma reflexão das possibilidades de intervenção por meio da História da Matemática como recurso didático. Os participantes foram instigados a refletir sobre a inserção do instrumento histórico nas aulas de matemática, uma vez que alguns já lecionavam e puderam resgatar suas próprias experiências em sala.

A princípio os alunos pesquisados relataram que não abordavam a História da Matemática e nem instrumentos matemáticos em sala de aula. Isto ocorre pela falta de domínio acerca da história do conteúdo, mas que o curso proposto seria uma forma de adquirir o conhecimento sobre a história dos Logaritmos e do instrumento Régua de Cálculo Circular. Essa realidade pode ser percebida na fala:

Durante minha graduação cursei a disciplina de História da Matemática, porém eu nunca relacionei com a minha prática em sala de aula, devido à insegurança de repassar conhecimentos que eu acho que não tenho. (PARTICIPANTE A)

No momento de discussão, os participantes relataram que o debate ampliou os conhecimentos sobre a história dos Logaritmos e a importância de instrumentos matemáticos históricos, permitindo que os mesmos pudessem introduzi-los durante sua experiência docente, concluindo que a História da Matemática é possível de inserção em sala de aula. Outro ponto mencionado é que o uso do instrumento como recurso didático, possibilita aprender de forma mais prática os conceitos e justificar sua existência.

A Régua de Cálculo Circular pode fornecer uma melhor perspectiva sobre o 
conceito, para ajudar a sedimentar e para servir como aula de exercícios (para não serem só exercícios rotineiros). (PARTICIPANTE B)

Desta forma, o conhecimento adquirido durante o curso pôde ser expandido para que os professores em formação construam conceitos de outros conteúdos matemáticos, visto que eles experimentaram em prática esse pensamento, realizando atividades na qual eles construíram outros tipos de régua. Esse caminhar que envolve o saber e o fazer já é estudado por Saito (2013, p. 105) reafirmando que "os instrumentos matemáticos podem, dessa maneira, revelar conhecimentos na articulação entre o saber e o fazer e, assim, a produção de conhecimento de uma época. Conhecimento este que pode receber diferentes interpretações e, consequentemente, significados".

O instrumento vislumbrado tanto pelos participantes como por Saito (2013) pode ser um agregador de vários saberes, como mostra a figura a seguir:

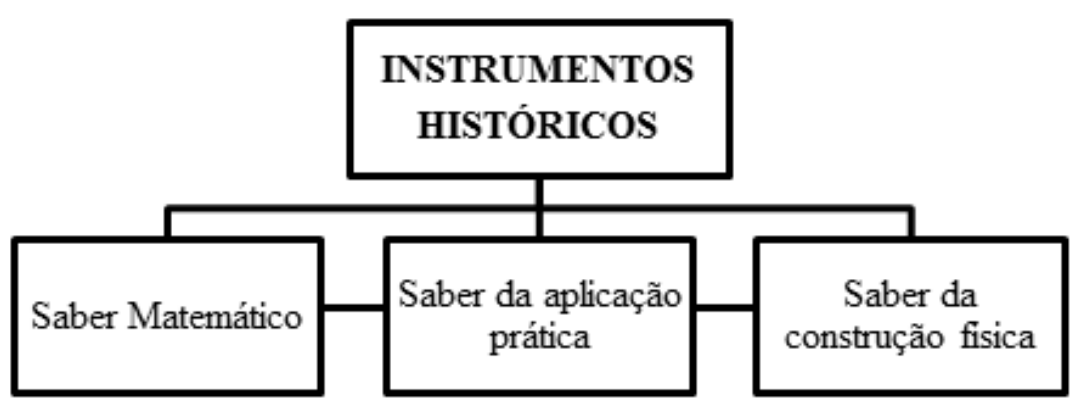

Figura 4 - Saberes Estudados nos Instrumentos Históricos

Fonte: Das autoras

Outro posicionamento dos participantes está relacionado ao contexto social, político, econômico e cultural da época. O estudo da história dos Logaritmos atrelado à construção da Régua de Cálculo Circular envolve a prática social do século XVII e com isso problematiza com a sociedade atual podendo formar uma Unidade Básica de Problematização - UBP que segundo Miguel e Mendes (2010, p. 386) é:

Um flash discursivo memorialístico que descreve uma prática social situada em um determinado campo de atividade humana, realizada para se responder a uma necessidade (ou desejo) que teria se manifestado por um ou mais indivíduos dessa comunidade, em algum momento do processo de desenvolvimento dessa atividade humana. (Tradução nossa).

A prática social pode ter diversos significados, dependendo da comunidade escolhida para aplicação. A UBP faz um resgate de uma prática Matemática histórica para 
o presente em forma de problematizações, trazendo para o processo de aprendizagem da Matemática a cultura, historicidade e simplicidade, de uma forma crítica-reflexiva. Sendo assim, a UBP é um convite ao ato do pensar.

Os posicionamentos podem ser analisados como potencialidades pedagógicas da Régua de Cálculo Circular. Em síntese percebe-se que:

- A Régua de Cálculo Circular é uma base para a construção de outros conceitos além dos Logaritmos, como por exemplo, no momento da construção, o professor aborda conteúdos geométricos;

- Há uma complementação do estudo dos Logaritmos por meio da Régua de Cálculo Circular;

- O conhecimento gerando instrumentos práticos (que evoluíram ao longo do tempo) que culminaram na criação dos nossos instrumentos atuais;

- O uso dos Logaritmos para obtenção de resultados é simples e direto quando utilizada a Régua de Cálculo Circular para o mesmo;

- Faz uso da prática, buscando na história da origem do conceito justificativas matemáticas para a existência do mesmo;

- Cenário para a construção de um problema que envolve a prática social de uma dada época (Unidade Básica de Problematização - UBP).

Durante a construção algumas dificuldades foram apresentadas pelos participantes com relação ao manuseio dos instrumentos para desenho, tais como o compasso e o transferidor. Esta dificuldade repercutiu no atraso da confecção da régua no momento da marcação da escala na régua de cálculo por meio do transferidor. Tais dificuldades provavelmente são decorrentes da escassez de conhecimento geométrico que não foi adquirido no decorrer da sua formação inicial. Por isso, cursos como esse são importantes, pois ampliam o conhecimento desses futuros professores e assim, tais dificuldades, que os alunos das escolas possivelmente também teriam, podem ser superadas.

\section{Considerações finais}

A partir das discussões no curso de extensão, utilizar a Régua de Cálculo Circular para o ensino dos Logaritmos se mostrou válida, sendo bem aceita pelos participantes.

Com o entendimento claro sobre o instrumento, o professor passa a enxergar melhor as possibilidades envolvidas, compreendendo que o mesmo pode ser confeccionado para outros fins, utilizando outros conteúdos matemáticos. Com isso, 
entendemos que aplicar a Régua de Cálculo Circular em sala de aula é um recurso auxiliador, não somente para o ensino de Logaritmos, mas também para outros conteúdos matemáticos, como os pertencentes a Geometria utilizados no momento da construção.

Quando se trata de aplicação para o conteúdo, a Régua de Cálculo Circular oferece ao aluno a possibilidade de desenvolver um novo olhar sobre toda álgebra que é apresentada em sala de aula. Essa aplicação favorece o ensino, permitindo um esclarecimento a respeito de um material precursor aos instrumentos de cálculo atuais.

Percebe-se ainda que a construção e o manuseio do instrumento fornecem a possibilidade de explorar. Quando o aluno se depara com esse tipo de atividade, pode tirar suas próprias conclusões sobre o conteúdo e investigar cada erro na construção, propiciando a busca por respostas individual ou coletivamente.

Portanto, a utilização da Régua de Cálculo Circular para o ensino dos Logaritmos é uma opção viável na qual os objetivos podem ser adequados às necessidades da turma, tais como explorar a aplicação dos Logaritmos, uma nova visão do conceito, a expansão das ideias, a criatividade do aluno quando se trata da construção e a compreensão do fazer matemático dos antigos.

Deixamos claro que para a utilização do instrumento, sugerimos um estudo prévio a respeito do material que o professor deseja explorar, pois assim como qualquer recurso a ser utilizado em sala de aula, o docente deve preparar-se para que não venha a obter resultados não esperados, como por exemplo, a complicação do conteúdo e o não entendimento por parte dos alunos. Portanto, o professor explorar a História da Matemática em suas aulas e a utilização de um instrumento histórico para fins didáticos é possível e traz vantagens no processo de ensino e aprendizagem.

\section{Referências}

FONSECA, Paulo Henrique Souza; PEREIRA, Ana Carolina Costa. A RÉGUA DE CÁLCULO E SEU POTENCIAL NO ENSINO DE CONTEÚDOS MATEMÁTICOS. In: XXII SEMANA DE EDUCAÇÃO DA UECE, 22., 2015, Fortaleza. Anais... Fortaleza: Uece, 2015. p. 1-8.

FROTA, Maria Clara Rezende. Experiência Matemática e investigação Matemática. In: CONGRESSO IBERO-AMERICANO DE EDUCAÇÃO MATEMÁTICA, 5., 2005, Porto. Anais... . Porto: Universidade do Porto, 2005. p. 1-10. Disponível em: <http://www.matematica.pucminas.br/Grupo de Trabalho/Maria clara/experiência Documento do Acrobat.pdf>. Acesso em: 27 abr. 2016.

LORENZATO, Sergio. Para aprender Matemática. Campinas: Autores Associados, 
2006. (Coleção formação de professores).

MAIA, Flávio do Sacramento. A régua de cálculo: uma aplicação das propriedades dos logaritmos. In: ENCONTRO NACIONAL DE EDUCAÇÃO MATEMÁTICA, 11. 2013, Curitiba. Anais... . Curitiba: Sbem, 2013. p. 1-14.

MENDES, Iran Abreu. Tendências Metodológicas no Ensino de Matemática. 1. ed. Belém: EDUFPA, 2008. v. 1.71p.

MIGUEL, Antonio; MENDES, Iran Abreu. Mobilizing histories in mathematics teacher education: memories, social practices, and discursive games. In: ZDM Mathematics Education (2010) 42, p. 381-392.

PEREIRA, Ana Carolina Costa. Aspectos Históricos da régua de cálculo para a construção de conceitos matemáticos. São Paulo: Livraria da Física, 2015. (História da Matemática para o Ensino).

PEREIRA, Maria Isabel da Costa; OLIVEIRA, José Damião Souza de. Da origem dos logaritmos ao uso da régua de cálculo no ensino de Matemática. In: ENCONTRO NACIONAL DE EDUCAÇÃO MATEMÁTICA, 11., 2013, Curitiba. Anais... • Curitiba: Sbem, 2013. p. 1-12.

PIPPA, Tania Cristina Maggioni. A função logaritmo e a régua de cálculo. 2014. 62 f. Dissertação (Mestrado) - Curso de Mestrado Profissional em Matemática em Rede Nacional, Universidade de São Paulo, São Carlos, 2014.

PORTELA, Maria Helena Pinheiro; FLORÊNCIO, Marianne Santos; MARTINS, Eugeniano Brito. Régua de cálculo: um resgate Histórico e sua aplicação na Matemática. In: FEIRA NACIONAL DA MATEMÁTICA, 3, 2014, Salvador. Anais ... Salvador, EDUNEB, 2014.

SAITO, Fumikazu. Instrumentos e o "saber-fazer" matemático no século XVI. Revista Tecnologia e Sociedade, Curitiba, v. 18, n. especial, 2013, p. 101-112.

SAITO, Fumikazu. Instrumentos matemáticos dos séculos XVI e XVII na articulação entre história, ensino e aprendizagem de Matemática. Rematec: História de Práticas Matemáticas, Natal, v. 16, n. 9, p.25-47, maio/ago. 2014.

TANONAKA, Elisa Missae. Régua de Cálculo: uma contribuição de William Oughtred para a Matemática.2008. 110 f. Dissertação (Mestrado) - Curso de Matemática, Pontifícia Universidade Católica de São Paulo, São Paulo, 2008. 